\title{
The Optimization of Automated Container Terminal Scheduling Based on Proportional Fair Priority
}

\author{
Yiqin Lu $(10$ \\ School of Economics and Management, Shanghai University of Electric Power, Shanghai, China \\ Correspondence should be addressed to Yiqin Lu; lu_yiqin@163.com
}

Received 8 October 2021; Accepted 28 December 2021; Published 18 January 2022

Academic Editor: Debiao Meng

Copyright $(2022$ Yiqin Lu. This is an open access article distributed under the Creative Commons Attribution License, which permits unrestricted use, distribution, and reproduction in any medium, provided the original work is properly cited.

\begin{abstract}
With the goal of maximizing the efficiency of automated container terminals, taking the loading and unloading time as the research object, the integrated optimization of automated container terminal scheduling with proportional fair priority is established by setting the priority parameters under the premise of balancing the operation efficiency of each container ship at the berth. The model considers separate assignments of general containers and special containers in the actual operation at the automated container terminal, which is never considered in other literatures. A proportional fairness algorithm (PFA) is designed to solve the problem, taking the maximum of the ratio of quantities of remaining loading and unloading containers of each container ship to the distance of empty AGVs/YTs as the proportional fair priority. The priority of all current tasks is ranked, and the highest priority task is fulfilled first. Different from the published literature, the study of this paper can be presented in two aspects. One is in the modeling. This includes (i) formalizing the description of the purpose of the model and (ii) conducting a real-world coordination of four types of equipment that incorporate at automated container terminals which considers different operations between general goods and special goods in the actual automated terminal operations. The other is that PFA is applied to deal with the integrated scheduling whose results are better with the balance of efficiency and fairness.
\end{abstract}

\section{Introduction and Literature Review}

As the largest automated container terminal in the world with the most advanced automatic technology, the highest level of intelligence, and zero green emissions, Shanghai Yangshan Automated Terminal adopts the automated terminal production control system and has been ranked the No. 1 in the world since 2010. The berthing time at the automated terminal of a container ship is determined directly by the loading and unloading efficiency of the automated terminal equipment. Thus, the research of the integrated scheduling of automated terminal equipment is of a great significance to improve terminal efficiency. The operation equipment at automated container terminal includes Quay Cranes (QCs), Automatic Guide Vehicles (AGVs), Automatic Stacking Cranes (ASCs), and Yard Trucks (YTs).

At present, domestic and foreign scholars have less research on the scheduling problem of automated container terminal equipment. Studies on the optimization of terminal operations are mainly focused on the following two aspects.

The first one is about the research on the optimization of traditional container terminal scheduling. Previous studies have mostly focused on the optimization of traditional container terminals, which also has a good reference for this paper. In traditional research, there were many studies on scheduling of common container terminals, most of which concentrated on the operation of QCs, YTs, and Yard Cranes (YCs) separately. Studies of isolated scheduling mainly covered distribution and scheduling of QCs [1-3], YC allocation and work sequences $[4,5]$, and scheduling and path optimization of YTs $[6,7]$. The joint optimization models were established for only two types of loading and unloading equipment including joint optimization of QCs-YTs [8], QCs-YCs $[9,10]$, and YT-YCs $[11,12]$. In addition, since these three operations affected each other, the three-stage integrated optimization of the operation system was the most effective means to improve efficiency from the 
perspective of system optimization [13]; Chen et al. [14], which was rare in previous literatures. When designing complex mechanical equipment, uncertainties should be considered to enhance the reliability of performance [15]. There are also many studies on optimization. Li et al. [16] realized the multiobjective optimization with a Modified Nondominated Sorting Genetic Algorithm III. A method of fuzzy optimization design-based multilevel response surface was proposed in a structural optimization design-based single-level response surface [17]. Finite element analyses are carried out, and the optimization scheme that minimizes the quality under the condition of satisfying the allowable stress is found [18].

The second aspect is about the research on optimization of the automated terminal operation system. Recent research on automated terminal horizontal transportation was mostly concentrated on the scheduling of AGVs [19-22]. The research on joint scheduling optimization of two kinds of operations includes joint scheduling of QC-AGV [23, 24] and joint scheduling of AGV-ASC [25]. To solve the cooperative scheduling of $\mathrm{AGV}-\mathrm{YC}$, the completing time under different conditions was obtained with the changes of AGVs, QCs, and container areas [25]. The abovementioned joint scheduling optimization limited to one or two types of equipment can only achieve optimal scheduling of the partial system rather than that of the entire system. As there are conflicts among the optimization objectives of the QCsAGVs-ASCs, only a few scholars have conducted relevant research on the optimization of the overall operation system of the automated container terminal scheduling. In order to realize the optimal scheduling of the whole system, Le and Zhang [26] proposed a new joint scheduling model of QCs, AGVs, and stacking cranes, which took the whole transportation system of the automated container terminal as the research object. Zeng and Yang [27] considered the cooperation problem of terminal transportation equipment and designed a multistage optimization algorithm. $\mathrm{Hu}$ and $\mathrm{Hu}$ [28] studied the coordinated scheduling mechanism among the three devices, proposed the full-degree-of-freedom scheduling problem of integrated shore bridges, stacking cranes, and YTs, and established a mixed integer programming model, but AGV was not considered in this study. Dkhil et al. [29] proposed the QC-AGV-ASC integrated model considering the interaction and constraints among various resources of the terminals. The above literatures showed that there were only a few studies on integrated scheduling of the automated container terminal scheduling. However, studies of the loading and unloading equipment and technology mentioned above are not mature enough and do not consider different operations between general goods and special goods which exist in the actual automated terminal operations. Since AGVs cannot transport ultralong, ultrawide, and dangerous containers, these two types of special containers are transported to special yards by the queued YTs at the berth. Therefore, the integrated optimization of scheduling at automated container terminals discussed in this paper will be more comprehensive, containing not only QCs, AGVs, and ASCs but also YTs, which is never considered in other literatures.
Based on the previous research $[30,31]$, the innovations of this paper are as follows: One is in the modeling. This includes (i) formalizing the description of the purpose of the model. The objective is to reduce the total operating time of all seaside ships while considering the operation time control of each ship; (ii Conducting a real-world coordination with proportional fair priority of four types of equipment that incorporate at automated container terminals which considers different operations between general goods and special goods. AGVs and YTs transport standard containers and special containers, respectively, and the operation difference between AGVs and YTs at the seaside is performed by double-trolley QCs. The other is that PFA is applied to deal with the integrated problem whose results are better with the balance of efficiency and fairness. Comparing with the Greedy Algorithm (GA), which is commonly used in optimization problems, PFA improves the overall efficiency by $5 \%$, and the fairness of loading and unloading time of each container ship is also greatly improved.

The paper is organized as follows. The problems are described and formulated in Section 2 and Section 3, respectively, and the model is proposed in Section 3. Section 4 presents the proportional fairness algorithm. Numerical experiments and results analysis are shown in Section 5 followed by conclusions in Section 6 .

\section{Problem Description and Assumptions}

Usually, the automated production control system is adopted as the "brain" to control the automatic operation system of the loading and unloading equipment, which consists of three types of loading and unloading equipment, namely, Quay Cranes, Automatic Guide Cranes, and Automatic Stacking Cranes. Figure 1 shows the single-container operation process at the automated terminal. The loading and unloading operation is completed by the double-trolley QCs. The main trolley is controlled remotely, and the portal trolley works automatically. They interact through the transfer platform. The horizontal transportation is finished by AGVs. The assignment of the yard is operated by ASCs which are fully automated. Three types of equipment work together. The seaside ASCs can complete doublecontainer operation. The YTs enter the berth, arriving at the designed container area. The land-side ASCs complete the unloading operation and go to the container yard. The export operation is carried out by the seaside ASCs. After the AGVs get the containers, they go to the bottom of the QCs. The automatic portal trolley hoists the containers to the transfer platform. The main trolley lifts the containers for loading and unloading operation. Thus, the entire export operation is completed. The import operation is the opposite operation process.

This paper takes the loading and unloading operation as the research object, adopts the principle of efficiency priority with consideration to fairness, and balances the containers to be transported of each ship as the constraint condition. A scheduling scheme to maximize the operational efficiency of the automated terminal is established by setting the priority parameters. In the integrated optimization model, QCs, 


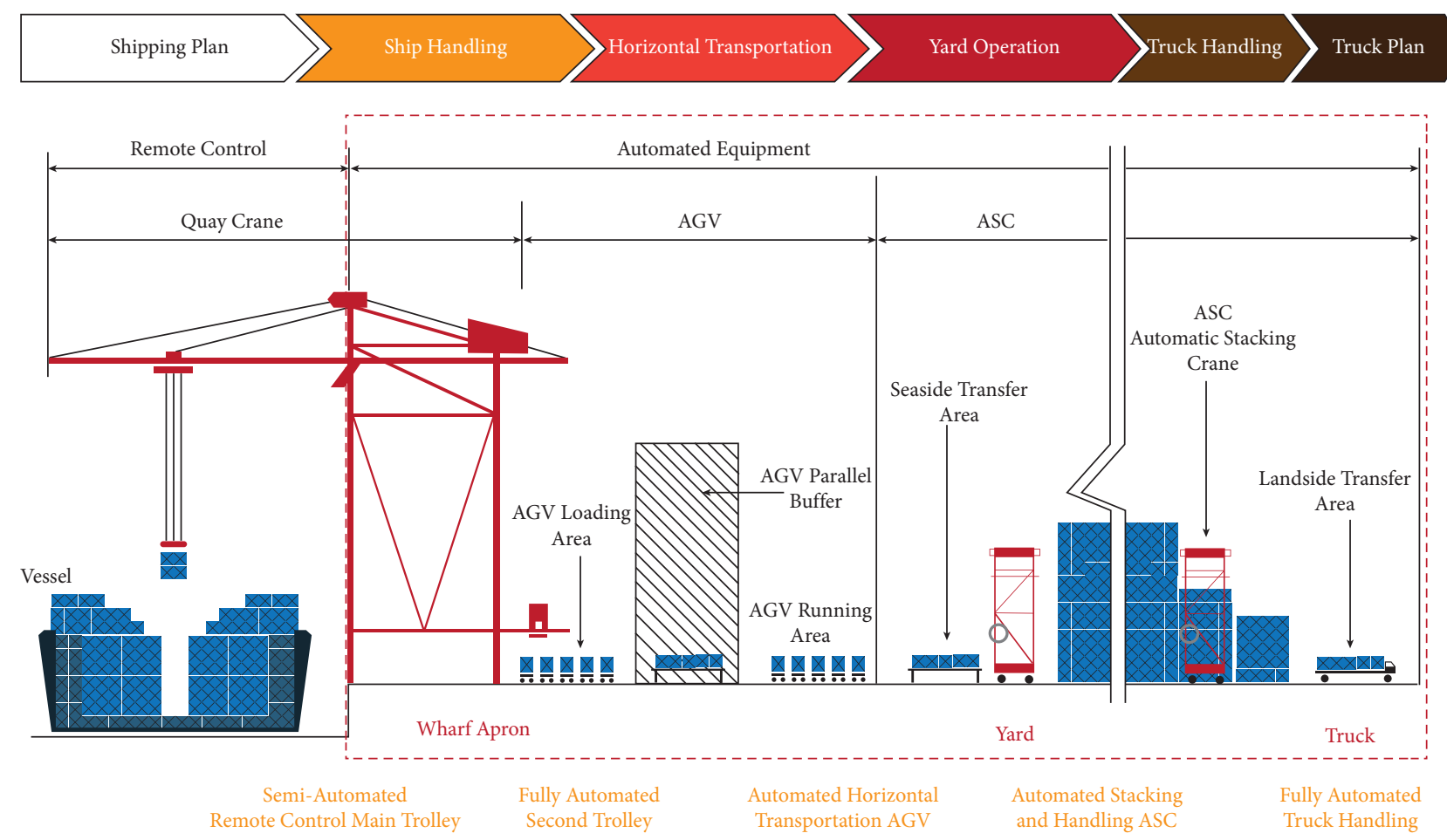

FIgURE 1: Single-container operation flow.

AGVs (or YTs), and ASCs cooperate with each other to maximize quantities of the loading and unloading containers per unit time, and the optimal scheduling plan of equipment scheduling is established considering the remaining container ships queued at the berth. The AGVs cannot deliver ultralong and ultrawide containers. Meanwhile, the automated container yards cannot stack these two kinds of nonstandard containers. Thus, they are transferred by YTs to the over-limit container yards. In addition, due to the related regulations, dangerous containers cannot be stored in the general container yards, so they should be transported by YTs to dangerous cargo yards. When the ship arrives, the general containers are transported by the portal trolley to the transfer platform for interaction and then transported by AGVs to the general cargo yard. The yard operation is completed by ASCs. Special containers, which are oversized or dangerous containers, will be transported to the special yards by queued YTs at the berth, not the AGVs. Figure 2 shows a full view of the automated terminal operation process. Therefore, making full use of the advantages of the double-trolley QCs to maximize the operational efficiency of the automated container terminal, the optimization model considered in this paper can satisfy different transportation requirements of all containers, not only general containers but also the other two special types of containers.

The optimization can be accomplished based on the following four assumptions:

(1) All loading and unloading tasks are completed.

(2) The operation task and operation time of each container are known, and there is no sequence for each loading and unloading task.
(3) The speeds of AGV and YT are known, and the operation speeds of QC and ASC are also known.

(4) There are no path conflicts between AGV and YT driving routes (that is, the road is wide enough).

(5) Each free AGV will find the most efficient task operation, while considering the multiship operation tasks. The optimal operating principle of $\mathrm{AGV}$ is to perform the task with the shortest operating time.

\section{Notations and Model}

The collection parameters are as follows:

$K$ represents cargo handling tasks, $K=\{1,2, \ldots, k\}=$ $K_{1} \cup K_{2}$

$K_{1}$ represents general cargo handling tasks of AGVs $K_{2}$ represents special cargo handling tasks of YTs

$N$ is the set of AGVs and YTs, $N=\{1,2, \ldots, n\}=N_{1} \cup N_{2}$

$N_{1}$ is the set of AGVs

$N_{2}$ is the set of YTs

For $i \in N$, tasks $L_{i} \subseteq M$ are assigned to the corresponding YTs or AGVs to satisfy the following conditions:

$\begin{aligned} L_{i} \cap L_{j} & =\varnothing, i \neq j ; \text { (2) } \coprod^{\tau=1}{ }^{m} L_{i}=M, \bigsqcup_{\tau \in N_{1}} L_{i}=M_{1}, \\ \coprod_{\tau \in N_{2}} L_{i} & =M_{2}\end{aligned}$ $P$ is the set of the two trolleys of QC, $p \in P, p(t)=$ $p_{1}(t)+p_{2}(t)$

$Q$ is the set of ASCs, $q \in Q$ 
Defining time series $p(t)$ and $q(t)$ as follows:

$p(t)=\left\{\begin{array}{ll}1 & \text { equipment } P \text { is busy } \\ 0 & \text { equipment } P \text { is free }\end{array}\right.$ and

$q(t)= \begin{cases}1 & \text { equipment } Q \text { is busy } \\ 0 & \text { equipment } Q \text { is free }\end{cases}$

$M$ represents the tasks that need to be loaded and unloaded of container ship $l$

$F_{l}$ is the completing time of all the loading and unloading tasks of container ship $l, F_{l}=\max _{m \in M_{l}} f_{m}$

The decision variables are as follows:

When AGV or YT is assigned to complete task $m$, the completing time is $T_{m \text {-total }}=t_{1}+t_{2}+t_{\text {loading }}+t_{\text {transport }}+$ $t_{3}+t_{\text {unloading }}$

$t_{1}$ is the time when the vehicle arrives at the start point of the task

$t_{2}$ is the waiting time of vehicle for container loading, $t_{2}=\min _{\text {loading }} t_{\text {finish }}$

$t_{\text {loading }} / t_{\text {transport }} / t_{\text {unloading }}$ are given and $t_{\text {transport }}=\mathrm{d} / v$

$t_{3}$ is the waiting time of vehicle for container unloading,

$t_{3}=\min _{\text {unloading }} t_{\text {finish }}$

$S_{m}$ is the time when each container task starts

$f_{m}$ is the time when each container task finishes

The whole model is written as follows

$$
\begin{aligned}
& \operatorname{Min} \sum_{l=1}^{L} F_{l}, \\
& F_{l}=\max _{m \in M_{l}} f_{m}, \\
& f_{m}=S_{m}+T_{m \text {-total }}, \\
& T_{m-\text { total }}=t_{1}+t_{2}+t_{\text {loading }}+t_{\text {transport }}+t_{3}+t_{\text {unloading }}, \\
& t_{2}=\min _{t}\left\{t \geq t_{1}+s_{m} \mid p_{i}(t)=0\right\}-\left(s_{m}+t_{1}\right), \\
& t_{3}=\min _{t}\left\{t \geq f_{m}-t_{\text {unloading }} \mid p_{i}(t)=0\right\} \\
& \quad-\left(f_{m}-t_{\text {unloading }}\right) .
\end{aligned}
$$

Equation (1) represents the minimum time for each container ship to complete loading and unloading operations.

Equation (2) shows that the time of the container ship to complete tasks is the maximum value of the time of completing all loading and unloading tasks.

Equation (3) represents the time when each task is completed and is the start time and the time required to complete the task

Equation (4) expresses the time to complete the task, including the time for empty YT to the start of the task, loading waiting time, loading time, transporting time, unloading waiting time, and unloading time.
Equation (5) indicates the calculating method of loading waiting time.

Equation (6) indicates the calculating method of unloading waiting time.

\section{The Proportional Fairness Algorithm}

The key of PFA is how to determine the proportional fair priority. Considering the quantities of remaining loading and unloading tasks of each container ship and the driving distance of empty $\mathrm{AGV} / \mathrm{YT}$, the priority of all current loading and unloading tasks is ranked, and the task with the highest priority is fulfilled first. Thus, the balance between efficiency and fairness is achieved. The principle of the proportional fairness algorithm in selecting tasks is as follows:

$$
\min _{l} \frac{\min _{i \mathrm{IM}_{1}} \text { finishing time of task } i}{\text { remaining containers of } \operatorname{ship} l} .
$$

The flow chart of the algorithm (Figure 3) is as follows:

(1) Make the statistics of the remaining loading and unloading containers of all ships performing tasks at the current time $\mathrm{Ni}$.

(2) Each container ship will find the nearest empty AGV or YT when loading and unloading containers. Calculate the time for the vehicle to reach the starting point of loading and unloading tasks $\mathrm{Ti}$.

(3) Compute the proportional fair priority Pri $=\max (\mathrm{Ni})$ $\mathrm{Ti}$ ) and choose the task with the highest priority.

\section{Numerical Experiments and Results}

In this chapter, PFA is designed for a typical business of automated container terminal. Compared with GA, which is commonly used in optimization problems, the efficiency of two algorithms is compared. The relative gain of PFA will be given through the simulation of MATLAB. The orders of job selection of two algorithms are different. In the application of GA, I first randomly selected an unfinished loading and unloading task and then selected the nearest AGV or YT to serve it. In PFA, the principles of selecting tasks are as follows:

$$
\min _{l} \frac{\min _{i \mathrm{M}_{1}} \text { finishing time of task } i}{\text { remaining containers of } \operatorname{ship} l}
$$

5.1. Data of Numerical Experiments. Suppose there are 180 loading and unloading containers to be loaded and unloaded in the designated terminal area. Loading and unloading positions of all tasks are fixed, and the quantities of AGVs, YTs, QCs, ASCs, and related parameters are known. The dataset is shown in Table 1.

5.2. Solutions of Numerical Experiments. The traditional GA and the innovative PFA are used to calculate the above 


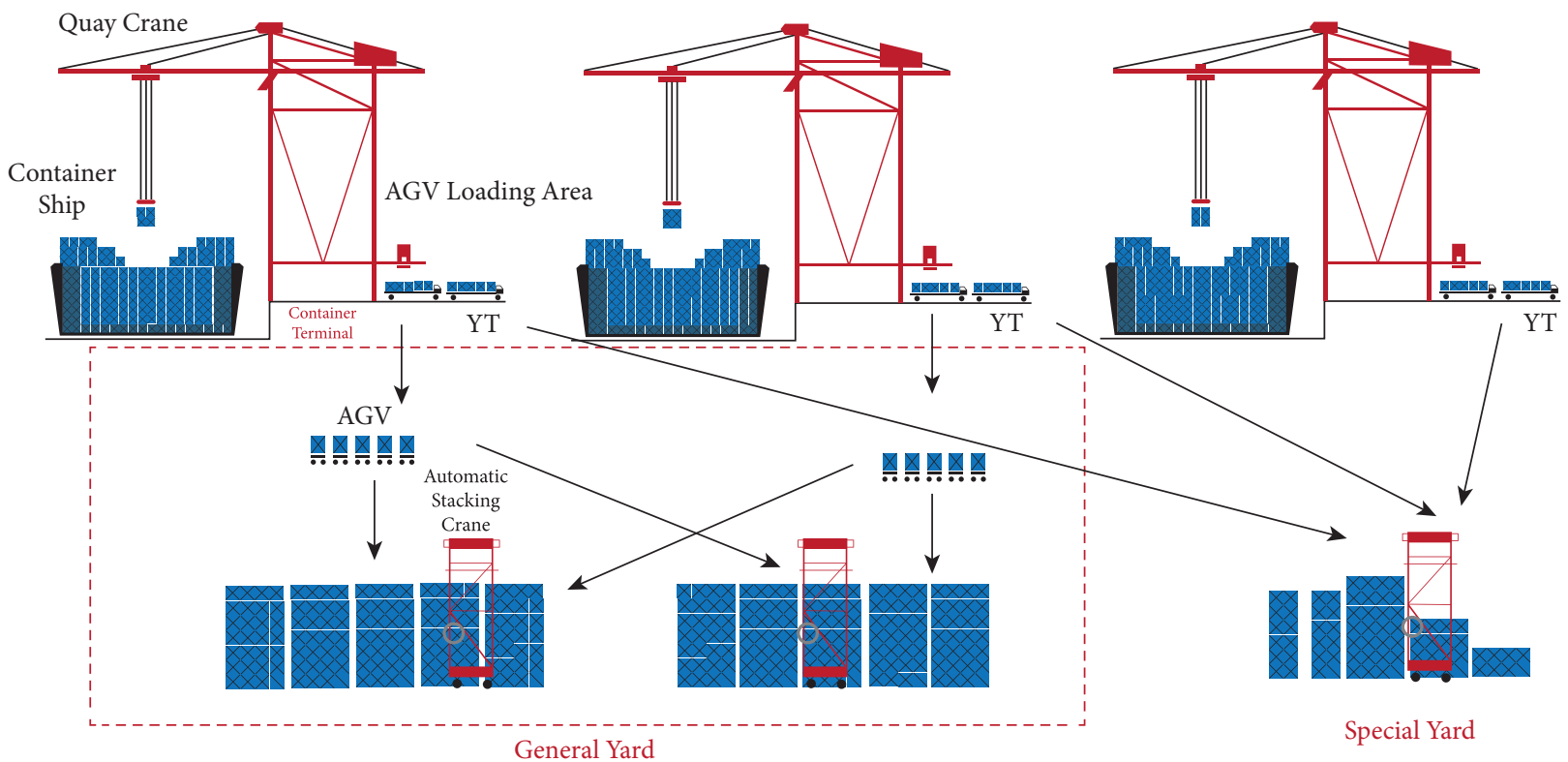

FiguRE 2: Full view of automated terminal operation process.

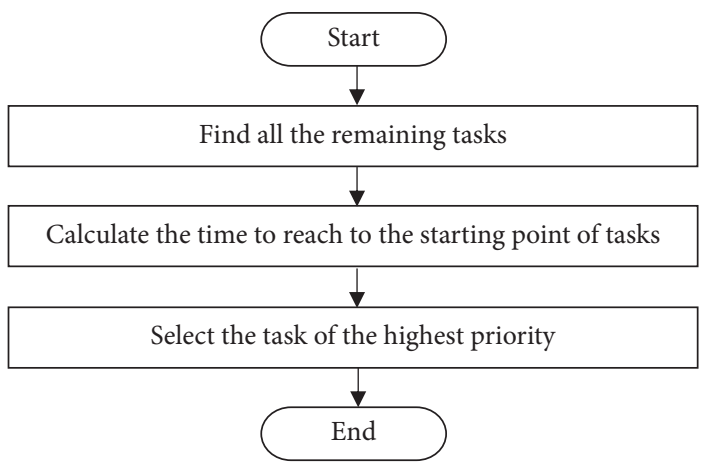

FIgURE 3: Flow chart of PFA.

TABLE 1: Dataset.

\begin{tabular}{lcc}
\hline Parameter & Number & Remarks \\
\hline Quantity of container ships & 4 & \\
Total loading containers & 92 & Quantities of loading containers by four ships are, respectively, 20, 40, 20, and 12 \\
Total unloading containers & 88 & Quantities of unloading containers by four ships are, respectively, 20, 20, 40, and 8 \\
Special containers & 18 & \\
Quantity of AGVs & 6 & \\
Quantity of YTs & 4 & \\
AGV speed & $30 \mathrm{~km} / \mathrm{h}$ \\
YT speed & $25 \mathrm{~km} / \mathrm{h}$ \\
Singer container operation time at the & 45 seconds \\
yard & 35 seconds \\
Singer QC operation time &
\end{tabular}

experiments, respectively. The results are shown in Table 2. The operation time of the four container ships of the two algorithms is listed separately. Taking the maximum value, the total operation time of GA is 1566 seconds, and that of PFA is 1479 seconds. Meanwhile, the standard deviation of operation time of four container ships is also listed in
Table 2. The advantages of PFA are obvious. From Table 2, the total operation time of PFA is increased by $5 \%$ compared with that of GA. Using the standard deviation to measure fairness, the standard deviation of PFA is reduced by $50 \%$ compared with that of GA. Thus, PFA has significantly improved the efficiency and fairness. The reason is that PFA 
TABLE 2: Completing time and standard deviation.

\begin{tabular}{lccccc}
\hline Completing time (second) & Container ship 1 & Container ship 2 & Container ship 3 & Container ship 4 & Standard deviation \\
\hline GA & 1523 & 1540 & 1524 & 1566 & 20.0728 \\
PFA & 1479 & 1461 & 1455 & 1460 & 10.50 \\
\hline
\end{tabular}

gives priority to loading and unloading operations with short operation time and more remaining tasks in task selection process.

\section{Conclusions}

As AGVs have limitations to transport over-limit containers and dangerous containers, these two special kinds of containers should only be transported by queued YTs to special cargo yards. In this context, the advantages of the doubletrolley QCs are fully utilized. By setting the priority parameters, the integrated model of QCs-AGVs (YTs)-ASCs scheduling is established under the premise of balancing the operating speed of each container ship at the berth. The model maximizes the efficiency of the automated terminal and solves the problem of fairness in operation of multiple container ships under various loading and unloading operations. PFA is designed to solve the problem, which is proved by numerical experiments with better efficiency and fairness. The integrated scheduling model helps to improve the efficiency of the automated terminals. In this paper, the choice of the task order of YT/AGV loading and unloading operation is studied. Future research will focus on scheduling at automated container terminals considering ASCQC loading and unloading task sequence.

\section{Data Availability}

The data including models, tables, and figures which are used to support the findings of this study are included within the article.

\section{Conflicts of Interest}

The author declares that there are no conflicts of interest.

\section{Acknowledgments}

This paper was supported by Humanities and Social Sciences Research Youth Fund Project of Education Ministry of China (18YJCZH116): Research on Integrated Optimization of Automated Container Terminal Scheduling under Uncertain Environment.

\section{References}

[1] P. Legato, R. Trunfio, and F. Meisel, "Modeling and solving rich quay crane scheduling problems," Computers \& Operations Research, vol. 39, no. 9, pp. 2063-2078, 2012.

[2] O. Uusal and C. Oguz, "Constraint programming approach to quay crane scheduling problem," Transportation Research Part E, vol. 59, pp. 108-122, 2013.

[3] J. H. Chen, D.-H. Lee, and M. Goh, "An effective mathematical formulation for the unidirectional cluster-based quay crane scheduling problem," European Journal of Operational Research, vol. 232, no. 1, pp. 198-208, 2014.

[4] Q. Q. Shao, Q. Xu, Z. Bian, and Z. H. Jin, "Stockpiling operating optimization for yard crane with containers delivery time uncertainty," Systems Engineering-Theory \& Practice, vol. 35, no. 2, pp. 394-405, 2015.

[5] D. Chang, Z. Jiang, W. Yan, and J. He, "Developing a dynamic rolling-horizon decision strategy for yard crane scheduling," Advanced Engineering Informatics, vol. 25, no. 3, pp. 485-494, 2011.

[6] Q. C. Zeng, X. J. Zhang, W. H. Chen, and X. C. Zhu, “Optimization model for truck appointment based on BCMP queuing network," Journal of Systems Engineering, vol. 28, no. 5, pp. 592-599, 2013.

[7] X. Guo, S. Y. Huang, W. J. Hsu, and M. Y. H. Low, “Dynamic yard crane dispatching in container terminals with predicted vehicle arrival information," Advanced Engineering Informatics, vol. 25, no. 3, pp. 472-484, 2011.

[8] C. Ma and C. J. Liang, "Integrated quay crane allocation and yard truck schedule problem in container terminals," Journal of Guangxi University(Natural Science Edition), vol. 40, no. 3, pp. 643-650, 2015.

[9] T. B. Qin, H. Ge, and S. Mei, "Unidirectional quay crane scheduling problems solving by combination of mixed integer programming and constraint programming," Computer Integrated Manufacturing Systems, vol. 21, no. 2, pp. 546-555, 2015.

[10] Y. Q. Xu and X. L. Han, "United scheduling model optimization of yard truck, quay crane and yard crane," Journal of Chongqing Jianzhu University, vol. 32, no. 2, pp. 318-320, 2013.

[11] Y. Wu, J. B. Luo, D. L. Zhang, and D Ming, “An integrated programming model for storage management and vehicle scheduling at container terminals," Research in Transportation Economics, vol. 42, no. 1, pp. 13-27, 2013.

[12] W. J. Mi, Y. F. Shen, and J. X. Y. X. M. Wang, "Collaborative decision-making on container pickup operation for gateway container selection and machinery resource allocation," Chinese Journal of Construction Machinery, vol. 12, no. 5, pp. 454-459, 2014.

[13] X. W. Xing, J. Mao, R. Zhang, and Z. H. Jin, "Optimization of container loading/unloading integrated scheduling in a container terminal based on hybrid flow shop," Chinese Journal of Management Science, vol. 22, no. 10, pp. 97-105, 2014.

[14] L. Chen, A.. Langevin, and Z. Q. Lu, "Integrated scheduling of crane handling and truck transportation in a maritime container terminal," European Journal of Operational Research, vol. 225, no. 1, pp. 142-152, 2013.

[15] D. B. Meng, Y. Li, S. P. Zhu, G. Lv, J. Correia, and Abilio de Jesus, "An enhanced reliability index method and its application in reliability-based collaborative design and optimization," Mathematical Problems in Engineering, pp. 1-10, 2019.

[16] Y. H. Li, Z. Sheng, P. Zhi, and D. Li, "Multi-objective optimization design of anti-rolling torsion bar based on modified 
NSGA-III algorithm," International Journal of Structural Integrity, vol. 12, no. 1, pp. 17-30, 2021.

[17] P. Zhi, Y. Li, B. Chen, M. Li, and G. Liu, "Fuzzy optimization design-based multi-level response surface of bogie frame," International Journal of Structural Integrity, vol. 10, no. 2, pp. 134-148, 2019.

[18] Y. H. Wang, C. Zhang, Y. Q. Su, L. Y. Shang, and T. Zhang, "Structure optimization of the frame based on response surface method," International Journal of Structural Integrity, vol. 11, no. 3, pp. 411-425, 2020.

[19] S. Y. Zhang, Y. S. Yang, C. J. Liang, B. W. Xu, and J. J. Li, "Optimal control of multiple AGV path conflict in automated terminals," Journal of Transportation Systems Engineering and Information Technology, vol. 17, no. 2, pp. 83-89, 2017.

[20] R. Zaghdoud and K. Mesghouni, "A hybrid method for assigning containers to AGVs in container terminal," IFACpapersOnLine, vol. 49, no. 3, pp. 96-103, 2016.

[21] J. B. Xin, "Control of interacting machines in automated container terminals using a sequential planning approach for collision avoidance," Transportation Research Part C: Emerging Technologies, vol. 60, pp. 377-396, 2015.

[22] J. B. Luo and Y. Wu, "Modeling of integrated vehicle scheduling and container storage problems in unloading process at an automated container terminal," Computers \& Industrial Engineering, vol. 94, pp. 32-44, 2016.

[23] C. Wei, Z. H. Hu, C. F. Gao, and X. J. Luo, "Scheduling model and algorithm of twin synchronized stacking cranes in stack yard of automated container terminal," Journal of Dalian Maritime University, vol. 41, no. 4, pp. 75-89, 2015.

[24] S. Y. Ma, Y. S. Yang, and C. J. Liang, "Collaborative scheduling of double-trolley quay cranes and AGV based on PSO at automated container terminal," Computer Applications and Software, vol. 35, no. 10, pp. 17-22, 2018.

[25] Y. S. Yang, Y. Y. Feng, C. J. Liang, B. W. Xu, and J. J. Li, "Integrated scheduling of automated guided vehicles and railmounted gantry cranes at automated container terminals," Journal of Shanghai Maritime University, vol. 38, no. 2, pp. 1-6, 2017.

[26] M. L. Le and Q. B. Zhang, "Integrated scheduling of quay cranes, automated guided vehicles and automated yard cranes at automated container terminals," Journal of Qingdao University of Science and Technology (Natural Science Edition), vol. 36, no. 5, pp. 569-574, 2015.

[27] Q. C. Zeng and Z. Z. Yang, "Integrating simulation and optimization to schedule loading operations in container terminals," Computers \& Operations Research, vol. 36, no. 6, pp. 1935-1944, 2009.

[28] S. L. Hu and Z. H. Hu, "Full freedom optimization for integrated quay cranes, Trucks and yard cranes in container port," Journal of Wuhan University of Technology, vol. 34, no. 5, pp. 58-63, 2012.

[29] H. Dkhil, A. Yassine, and H. Chabchoub, "Optimization of container handling systems in automated maritime terminal," Studies in Computational Intelligence, vol. 457, pp. 301-312, 2013.

[30] Y. Q. Lu and M. L. Le, "The integrated optimization of container terminal scheduling with uncertain factors," Computers \& Industrial Engineering, vol. 75, pp. 209-216, 2014.

[31] Y. Q. Lu, "The three-stage integrated optimization of automated container terminal scheduling based on improved genetic algorithm," Mathematical Problems in Engineering, vol. 2021, Article ID , 9 pages, 2021. 